\title{
DIALOGUE
}

\section{In defense of the frontal lobe hypothesis of cognitive aging}

\section{ROBERT WEST}

University of Notre Dame, Notre Dame, Indiana

(Received September 27, 1999; ACCEPTED October 1, 1999)
Over the past decade a number of reviewers have argued that the pattern of spared and impaired cognitive functions observed in the cognitive aging literature can be understood within the context of a neuropsychologically constrained model that proposes that the prefrontal cortex is more vulnerable to the effects of normal aging than other cortical regions (Dempster, 1992; Hartley, 1993; Moscovitch \& Winocur, 1992; West, 1996). While this model has served as a valuable heuristic in my own thinking about cognitive aging, the current review of Greenwood and theoretical work emerging from other laboratories argues that the "frontal lobe hypothesis" (FLH) no longer provides a useful or accurate framework within which to understand brain behavior relationships in cognitive aging. While I agree with several of the ideas developed in the review of Greenwood (this issue) I believe the continued utility of the FLH can be demonstrated by considering two questions: (1) What does the FLH predict about cognitive aging and is there support for these predictions? and (2) Has the time come to move beyond a general specification of the FLH? In answering these questions I will provide one or two findings from the empirical literature. These are intended to be illustrative, not exhaustive.

One of the strongest arguments against the FLH presented by Greenwood resides in the use of the term "selective" in relating age-related declines in cognitive function to the prefrontal cortex. I feel at least partially responsible for this concern as my 1996 paper opened with the following, somewhat bold, statement: "In the past decade, a rapidly expanding body of evidence from the field of geriatric neuropsychology has provided evidence suggesting a selective $^{1}$, age-related decrease in performance on neuropsychological measures diagnostic of frontal lobe insult (West, 1996, p. 272).

Reprint requests to: Robert West, 118 Haggar Hall, Department of Psychology, University of Notre Dame, Notre Dame, IN 46556. E-mail: robert.west.19@nd.edu

${ }^{1}$ Emphasis added by author.
This quote and the concern of Greenwood raise an interesting question. Does anyone really believe that age-related decline is selectively focused on the prefrontal cortex? While I cannot speak for other reviewers in this area, I can say with confidence that I have never held the opinion that the neurobiological sequelae of the aging process were limited to the prefrontal cortex. Instead, the FLH should be considered within the context of two assumptions that provided the foundation of the argument developed in West (1996):

1. Age-related declines in cognitive processes supported by the prefrontal cortex should emerge at an earlier age than age-related declines in cognitive processes supported by nonfrontal regions.

2. Age-related declines in cognitive processes supported by the prefrontal cortex should be greater in magnitude than age-related declines observed in cognitive processes supported by nonfrontal regions.

While neither of these assumptions has been extensively tested in the research literature there is empirical support for each. Work by Shimamura and Jurica (1994) has demonstrated that reliable age-related declines in the Self Ordered Pointing Task ( $M$ age 61 years) — associated with the prefrontal cortex in both humans (Petrides \& Milner, 1982) and primates (Petrides, 1995) - emerged earlier than reliable age-related declines in recognition memory ( $M$ age 71 years)-typically associated the hippocampal complex (Moscovitch \& Winocur, 1992). Complimenting this behavioral evidence, a longitudinal study of age-related changes in resting cerebral blood flow (rCBF) revealed significant reduction in $\mathrm{rCBF}$ for the prefrontal, but not motor, frontotemporal, occipital, or temporal regions over a 4-year period (Shaw et al., 1984). Together these findings provide support for the assumption that the prefrontal cortex - and associated cognitive processes-demonstrate signs of decline earlier than nonfrontal regions-and associated cognitive processes. 
Evidence consistent with the assumption that age-related declines in prefrontal cognitive processes are greater than declines observed in cognitive processes supported by other regions has been provided in both experimental and metaanalytic work. A number of studies incorporating the Stroop task have consistently demonstrated that the magnitude of the interference effect is greater for older than younger adults when color and word information are integrated at the same location, while the magnitude of the interference effect is comparable for younger and older adults when color and word are presented at spatially separate locations (Hartley, 1993; West \& Bell, 1997). Furthermore, West and Bell (1997) have demonstrated that this behavioral effect extends to patterns of cortical activation, as the level of alpha suppression in the EEG invoked by task performance is greater for older than younger adults over frontal and parietal regions for integrated but not separated stimuli. Hartley (1993) has suggested that this dissociation results from the differential reliance on the posterior-proposed to be largely immune to the effects of age-and anterior-proposed to be compromised as part of the aging process - attention systems to perform the Stroop task under conditions of color and word separation or integration, respectively. In essence, older adults are thought to rely on an intact posterior attention system to filter word information on separated trials, and be forced to rely on a compromised anterior attention system to deal with the interfering effect of the word on integrated trials. The findings from an extensive meta-analysis of the literature on memory and aging provides additional evidence that age-related declines in those aspects of memory often attributed to the prefrontal cortex (e.g., recall or recollection of the past; Nyberg et al., 1996) are greater than those aspects of memory believed to be supported by the occipital region (e.g., priming; Buckner et al., 1998; La Voie \& Light, 1994).

Another interesting proposal of Greenwood (this issue) is that differences observed between younger and older adults in recent cognitive activation studies using positron emission tomography are not consistent with the FLH. While I agree with Greenwood (this issue) that it is important to consider the effects of age on dynamic processing networks revealed in neuroimaging studies, I believe that the findings of such analyses have often been consistent with the FLH. A number of imaging studies have revealed differential involvement of the prefrontal cortex in older adults relative to younger adults in a variety of tasks (for a review, see Grady, 1999), consistent with the idea that advancing age is associated with a decline in the functional integrity of the prefrontal cortex.

A consistent finding to emerge from imaging studies that may initially be seen as troubling for the FLH is that the age-related decrease in activation of one frontal region is often accompanied by an age-related increase in the activation of another frontal region (Cabeza et al., 1997; Grady, 1998). I would argue that such findings do not necessarily violate the FLH, but instead reveal a need for the refinement of the hypothesis to allow for differential age-related decline within the prefrontal cortex and the cognitive operations supported by this region. The idea that there may be differential decline within the prefrontal cortex emerges from evidence across a number of sources. At the structural level a recent comparative analysis revealed some 18 different architectonic regions within the primate and human prefrontal cortex (Pandya \& Yeterian, 1998). Given the vast functional and architectural landscape of the prefrontal cortex it seems reasonable to believe that the effects of increasing age may not be uniform across this region. Supporting this idea, Haug and Eggers (1991) have reported that agerelated volume reduction was greater in the dorsolateral than orbital prefrontal regions. More recent work by Raz and colleagues (Raz et al., 1997) has revealed similar linear trends in the degree of age-related volume reduction in the dorsolateral and orbital regions, commensurate with a quadratic age-related trend unique to the orbital region. In addition to these structural data the findings of a recent event-related brain potential (ERPs) study also suggest that neurocognitive processes supporting performance of the Stroop task focused over the prefrontal cortex were differentially affected by the aging process (West \& Alain, 2000). In this study the amplitude of ERPs associated with conflict detection were markedly attenuated in older adults, while the amplitude of ERPs associated with the controlled processing of color information in older adults was slightly greater than that observed in younger adults. Together these data illustrate that it is no longer sufficient to speak of a general decline in cognitive processes supported by the prefrontal cortex, but has become necessary to explore the differential effects of aging on the variety of cognitive processes supported by this neural region.

Identifying the neurobiological foundations of age-related declines in cognitive function represents a challenging undertaking that requires the application of progressive methodological approaches from the areas of cognitive psychology, neuropsychology, neuroscience, and computational science. The current work of Greenwood demonstrates how the creative integration of empirical findings from across these various domains can be used to construct neurobiologically constrained models of cognitive aging. The task of future investigators will be to exercise equal creativity in designing multidisciplinary research programs that can provide prospective empirical tests of such models.

\section{REFERENCES}

Buckner, R.L., Goodman, J., Burock, M., Rotte., M., Koustaal, W., Schacter, D., Rosen, B., \& Dale, A.M. (1998). Functionalanatomic correlates of object priming in humans revealed by rapid presentation event-related fMRI, Neuron, 20, 285296.

Cabeza, R., Grady, C.L., Nyberg, L., McIntosh, A.R., Tulving, E., Kapur, S., Jennings, J.M., Houle, S., \& Craik, F.I.M. (1997). Age-related differences in neural activity during memory encoding and retrieval: A positron emission tomography study. Journal of Neuroscience, 17, 391-400. 
Dempster, F.N. (1992). The rise and fall of the inhibitory mechanism: Toward a unified theory of cognitive development and aging. Developmental Review, 12, 45-75.

Grady, C.L. (1998). Brain imaging and age-related changes in cognition. Experimental Gerontology, 33, 661-673.

Hartley, A.A. (1993). Evidence for the selective preservation of spatial selective attention in old age. Psychology and Aging, 8, 371-379.

Haug, H. \& Eggers, R. (1991). Morphometry of the human cortex cerebri and corpus striatum during aging. Neurobiology of Aging, $12,336-338$.

La Voie, D. \& Light, L.L. (1994). Adult age differences in repetition priming: Ameta-analysis. Psychology and Aging, 9, 539-553.

Moscovitch, M. \& Winocur, G. (1992). The neuropsychology of memory and aging. In F.I.M. Craik \& T.A. Salthouse (Eds.), The handbook of aging and cognition (pp. 315-372). Hillsdale, NJ: Lawrence Erlbaum Associates.

Nyberg, L., Cabeza, R., \& Tulving, E. (1996). PET studies of encoding and retrieval: The HERA model. Psychonomic Bulletin and Review, 3, 135-148.

Pandya, D.N. \& Yeterian, E.H. (1998). Comparison of prefrontal architecture and connections. In A.C. Roberts, T.W. Robbins, \& L. Weiskrantz (Eds), The prefrontal cortex: Executive and cognitive functions (pp. 51-66). New York: Oxford University Press.

Petrides, M. (1995). Impairments on nonspatial self-ordered and externally ordered working memory tasks after lesions of the mid-dorsal part of the lateral frontal cortex in the monkey. Journal of Neuroscience, 15, 359-375.

Petrides, M. \& Milner, B. (1982). Deficits on subject-ordered tasks after frontal- and temporal-lobe lesions in man. Neuropsychologia, 3, 249-262.

Raz, N., Gunning-Dixon, F.M., Head, D., Dupuis, J.H., McQuain, J., Briggs, S.D., Loken, W.J., Thornton, A.E., \& Acker, J.D. (1997). Selective aging of the human cerebral cortex observed in vivo: Differential vulnerability of the prefrontal gray matter. Cerebral Cortex, 7, 268-282.

Shaw, T.G., Mortel, K.F., Meyer, J.S., Rogers, R.L., Hardenberg, J., \& Cutaia, M.M. (1984). Cerebral blood flow changes in benign aging and cerebrovascular disease. Neurology, 34, 855-862.

Shimamura, A.P. \& Jurica, P.J. (1994). Memory interference effects and aging: Findings from a test of frontal lobe function. Neuropsychology, 8, 408-412.

West, R.L. (1996). An application of prefrontal cortex function theory to cognitive aging. Psychological Bulletin, 120, 272-292.

West, R. \& Alain, C. (2000). Age-related decline in inhibitory control contributes to the increased Stroop effect observed in older adults. Psychophysiology, 37, 179-189.

West, R. \& Bell, M.A. (1997). Stroop color-word interference and electroencephalogram activation: Evidence for age-related decline in the anterior attention system. Neuropsychology, 11, 421427. 